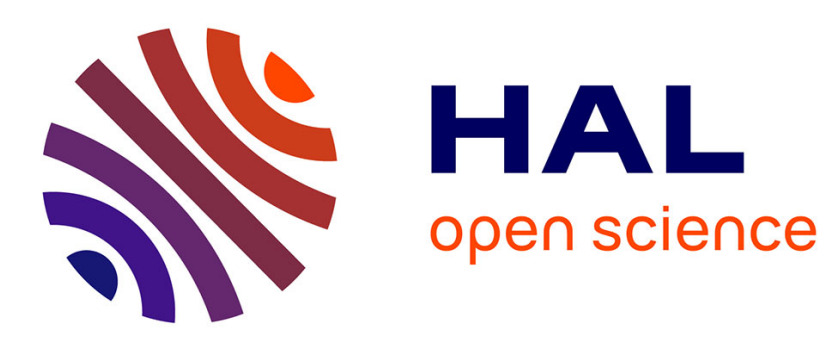

\title{
A multiresolution wavelet scheme for irregularly subdivided 3D triangular mesh
}

Sébastien Valette, Yun-Sang Kim, Ho-Youl Jung, Isabelle Magnin, R. Prost

\section{To cite this version:}

Sébastien Valette, Yun-Sang Kim, Ho-Youl Jung, Isabelle Magnin, R. Prost. A multiresolution wavelet scheme for irregularly subdivided 3D triangular mesh. 6th International Conference on Image Processing (ICIP'99), 1999, Kobe, Japan. pp.171-174. hal-02272216

\section{HAL Id: hal-02272216 https://hal.science/hal-02272216}

Submitted on 28 Aug 2019

HAL is a multi-disciplinary open access archive for the deposit and dissemination of scientific research documents, whether they are published or not. The documents may come from teaching and research institutions in France or abroad, or from public or private research centers.
L'archive ouverte pluridisciplinaire HAL, est destinée au dépôt et à la diffusion de documents scientifiques de niveau recherche, publiés ou non, émanant des établissements d'enseignement et de recherche français ou étrangers, des laboratoires publics ou privés. 


\title{
A multiresolution wavelet scheme for irregularly subdivided 3D triangular mesh
}

\author{
Sébastien Valette ${ }^{l}$, Yun-Sang Kim ${ }^{l}$, Ho-Youl Jung ${ }^{2}$, Isabelle Magnin ${ }^{l}$ and Rémy Prost ${ }^{l}$ \\ ${ }^{1}$ CREATIS, CNRS Research Unit (UMR 5515) and affiliated to INSERM, INSA, Villeurbanne, France \\ ${ }^{2}$ School of Computer \& Communications Eng., Yeungnam University, Kyungpook, Korea \\ E-mail: \{sebastien.valette, yun-sang.kim,remy.prost\}@creatis.insa-lyon.fr, \\ hoyoul@ynucc.yeungnam.ac.kr
}

\begin{abstract}
We propose a new subdivision scheme derived from the Lounsbery's regular 1:4 face split, allowing multiresolution analysis of irregularly subdivided triangular meshes by the wavelet transforms. Some experimental results on real medical meshes prove the efficiency of this approach in multiresolution schemes. In addition we show the effectiveness of the proposed algorithm for lossless compression.
\end{abstract}

\section{Introduction}

Multiresolution analysis of 3D objects is receiving a lot of attention nowadays, due to the practical interest of 3D modelling in a wider and wider range of applications. Multiresolution analysis of these objects gives some useful features : several levels of details can be built for these objects, accelerating the rendering when there is no need for sharp details, and allowing progressive transmission. Another feature is that multiresolution analysis can be an efficient way for data compression. A survey of the existing methods used to simplify meshes which is the first step for processing multiresolution analysis, like vertex decimation [2], edge contraction [3] and wavelet surfaces [4], was reported in [1]. We put our attention on the third method, because wavelets are wellsuited for multiresolution analysis. In section 2, we will shortly explain multiresolution analysis of meshes [3], and show its drawbacks in practical implementation, which we improved, as described in section 3 . In section 4 , we show why our proposal is suitable for compression. The next part (section 5) gives the results obtained with this new scheme.

\section{Lounsbery's wavelets based multiresolution scheme}

In wavelets decomposition, a mesh (for example a tetrahedron, see figure 1.a) is quaternary subdivided (figure 1.b) and deformed (figure 1.c), to make it fit the surface to approximate. Subdividing the mesh consists in splitting each triangular face into four faces. These steps can be processed depending on the required resolution levels.

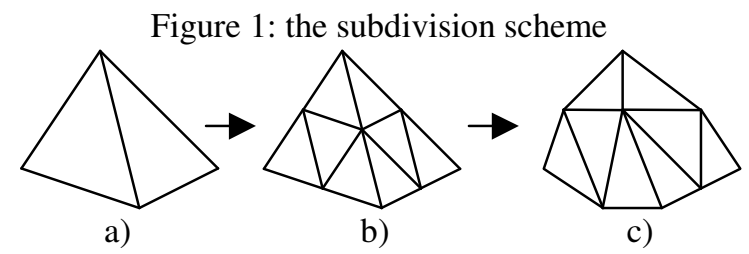

Multiresolution analysis is computed with two analysis filters $A^{j}$ and $B^{j}$ for each resolution level $j$. Reconstruction is done with two synthesis filters $\mathrm{P}^{\mathrm{j}}$ and $\mathrm{Q}^{\mathrm{j}}$. These filters are represented with matrix notation and, to ensure exact reconstruction, must satisfy the following constraint:

$$
\left[\frac{A^{j}}{B^{j}}\right]=\left[P^{j} \mid Q^{j}\right]^{-1}
$$

Let us call $C^{i}$ the $N(j) \times 3$ matrix giving the coordinates of each vertex of the mesh at the resolution level $\mathrm{j}$. Then we have the relations:

$$
\begin{gathered}
C^{\mathrm{j}}=\mathrm{A}^{\mathrm{j}+1} \cdot \mathrm{C}^{\mathrm{i}+1} \\
\mathrm{D}^{\mathrm{j}}=\mathrm{B}^{\mathrm{j}+1} \cdot \mathrm{C}^{\mathrm{j}+1} \\
\mathrm{C}^{\mathrm{j}+1}=\mathrm{P}^{\mathrm{j}} \cdot \mathrm{C}^{\mathrm{j}}+\mathrm{Q}^{\mathrm{j}} \cdot \mathrm{D}^{\mathrm{j}}
\end{gathered}
$$

$D^{j}$ represents the wavelet coefficients of the mesh, necessary to reconstruct $\mathrm{C}^{\mathrm{j}+1}$ from $\mathrm{C}^{\mathrm{j}}$. From a theoretical point of view, each column of the $\mathrm{P}^{\mathrm{j}}$ matrix (respectively the $Q^{j}$ matrix) represents a scaling function (respectively a wavelet function). These functions are defined on a 3D space fixed by the mesh topology. 
We apply the lifting scheme [6] which consists here in constructing wavelet functions (starting from the hat function, figure 2.a) orthogonal to the scaling functions (which are hat functions too, but with a twice wider support). Without the lifting scheme, Lounsbery's multiresolution analysis would simply consist in subsampling the mesh, but with the lifting, the mesh at resolution level $\mathrm{j}$ is ensured to be the best approximation in the mean square sense for the mesh at level $j+1$. The main material for the lifting is the inner product between two functions defined by Lounsbery as:

$$
<f, g>=\sum_{\tau \in \Delta(M)}\left(\frac{K_{j}}{\operatorname{area}(\tau)} \int_{s \in \tau} f(s) \cdot g(s) \cdot d s\right)
$$

$\Delta(\mathrm{M})$ is the set of triangles $\tau$ of the mesh and $\mathrm{K}_{\mathrm{j}}$ is a constant for a given resolution level $\mathrm{j}$. $\left(\mathrm{K}_{\mathrm{j}}=4^{-\mathrm{j}}\right)$. Note that in this inner product it is assumed that the triangular faces of the mesh have the same area. The consequence of this assumption is that a mesh at resolution level $\mathrm{j}$ will effectively be the best approximation of the mesh at level $j+1$ only if this constraint is fulfilled. We can see in figure 2.b) the effects of the lifting scheme on the hat function showed in figure 2.a).

Figure 2: wavelets in 3D a)

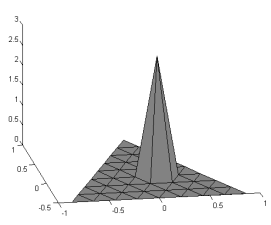

b)

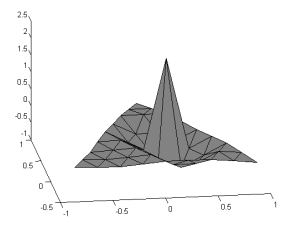

Wavelet surfaces give a powerful tool for multiresolution analysis. However, in the simplification process, the major drawback is that faces are always merged four to one to have a simpler mesh. If the mesh does not respect this connectivity constraint, one has to process a resampling of the mesh, which results in a mesh having more faces than the original, as explained in details in [5]. The aim of this work is to solve this problem by improving the subdivision process, as described in the next section.

\section{A proposal for irregular subdivision}

In the proposed scheme, the subdivision process is changed : each face of the mesh to subdivide is no more systematically split into four faces, but can also be split into three or two faces or remain unchanged. As an example, four different cases of subdivision are shown in figure 3 . This approach allows to simplify meshes even if some faces cannot be merged four to one.
Figure 3: some possible cases of subdivision

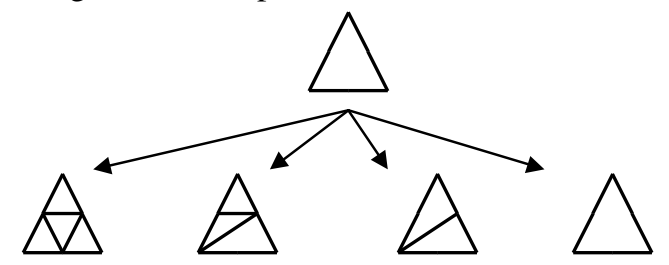

The simplification is done with an algorithm that merges the faces of a mesh, considering rules established by the subdivision process. Figure 4 shows an example, where 15 faces are reduced to 6 , resulting from merging $4: 1$ faces for $\mathrm{G} 2,3: 1$ faces for G3 and G6, $2: 1$ faces for $\mathrm{G} 1$ and G4 and keeping one face unchanged for G5.

Figure 4: an example of surface simplification
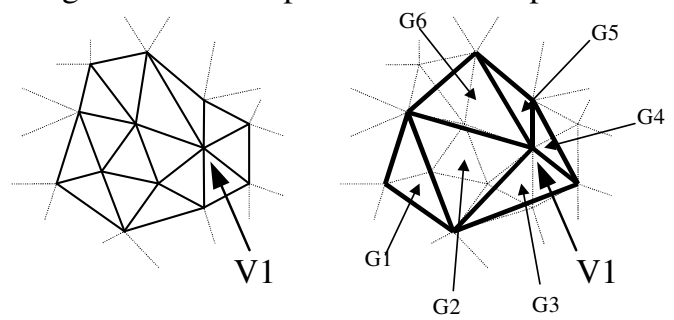

One important consequence of the simplification rules is that a vertex can be removed during the simplification process only if its valence is equal to 4,5 or 6 . Clearly the efficiency of our algorithm depends on the number of removable vertices in the mesh. Fortunately, meshes usually have such vertices, and during the simplification, the valence of the vertices tends to decrease. As an example, the vertex V1 shown in figure 4 has a valence of 7, which makes it impossible to remove, but after one simplification step, its valence is reduced to 5, and the algorithm will be able to remove it in a further step.

Briefly, the simplification algorithm starts by merging 4 faces to 1 , building a set of merged faces, and tries to expand this set by merging faces around it. Figure 5 shows the beginning of the expansion of the merged faces set (in gray), merging sequentially $4: 1$ faces, $3: 1$ faces and $2: 1$ faces.

Figure 5: expansion of the simplified mesh set a)

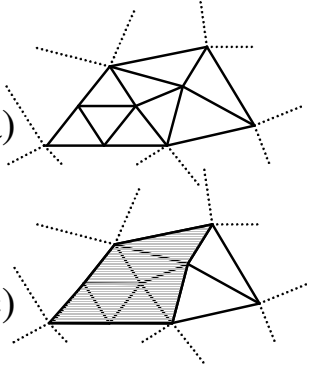

b)

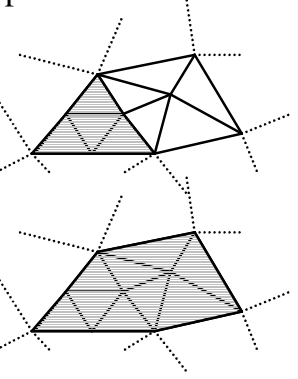


The algorithm stops when no more faces have to be simplified. In order to prevent the algorithm from being unable to simplify some faces with respect to the subdivision rules, a modification of the mesh is allowed. It consists in an edge permutation between two neighbour faces, as shown in figure 6 . Of course this modification has to be stored, to recover the original mesh after subdivision and guarantee the reversibility of the simplification process.

Figure 6: an edge permutation between two faces
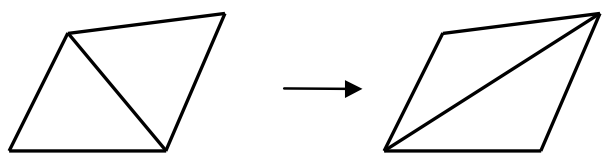

We notice that this modification will introduce a quality loss during multiresolution analysis, but the difference between the original mesh and the altered mesh is small and experimental results show that this local error is negligible compared to the approximation error. Finally, the algorithm is very efficient for simplifying a large set of meshes.

The last thing to do is to compute the approximation of the high resolution mesh with the simplified one that is to calculate the analysis filters $\mathrm{A}^{\mathrm{j}}$ and $\mathrm{B}^{\mathrm{j}}$. This can be done with Lounsbery's scheme. A difference has to be noticed, due to the change of the subdivision process. The inner product (5) has to be reformulated and becomes:

$$
<f, g>=\sum_{\tau \in \Delta(M)}\left(\frac{K_{j}(\tau)}{\operatorname{area}(\tau)} \int_{s \in \tau} f(s) \cdot g(s) \cdot d s\right)
$$

$\mathrm{K}_{\mathrm{j}}(\tau)$ is no longer a constant and changes with each face of the mesh. For example, a face in a low resolution mesh that will split in 3 faces will have $K_{j}(\tau)=3$ and the three resulting faces will have $K_{j+1}(\tau)=1$, taking into account the differences between the triangle areas : the first face cited above will approximately be three times larger than the three last.

\section{Compression}

The proposed method has powerful features for compressing meshes, for two reasons:

- The wavelet decomposition, used to compute the vertices coordinates, transforms coordinates into wavelet coefficients which histogram is concentrated around the zero value, making them well suited for entropy coding.
- Starting from the lowest resolution level, there is no need to store or transmit the faces descriptions to reconstruct higher levels, only the subdivisions have to be, which lets the amount of information needed to reconstruct the connectivity of the mesh close to 3 bits per face.

In the experimental results section, the lowest resolution mesh is coded using the algorithm described in [8].

\section{Results}

Table 1 shows the results on a heart mesh simplification using the proposed method. The high resolution mesh is a regular tesselation similar to that proposed in [7]. Its vertices coordinates are coded with a 7 bits/vertex precision. The number of bytes noted in table 1 is the amount of information needed to reconstruct the mesh to the concerned resolution level starting from the nearest lower resolution level.

Table 2 gives some more results on different 3D meshes: brain, body and lung. We notice that at the middle resolution levels, used in practical implementation, the approximation quality remains rather good in terms of local shape and size.

Table 1: multiresolution representation of a 3D left ventricle of a heart mesh

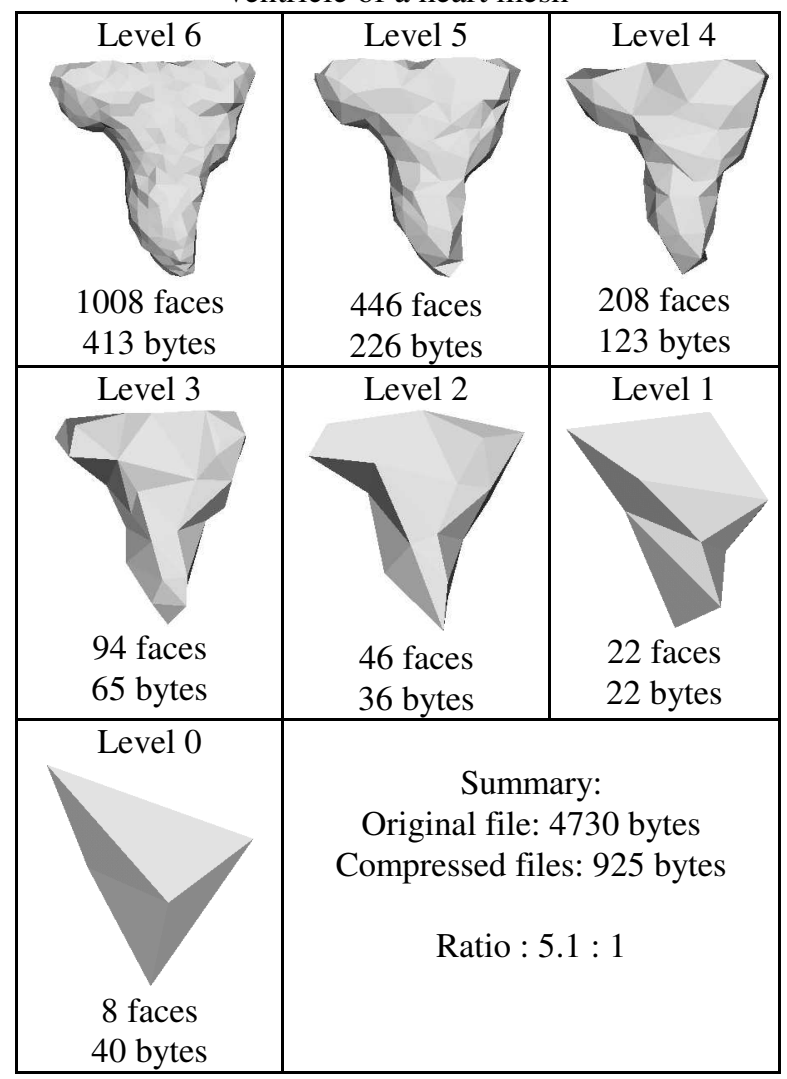


Table 2: complementary results

\begin{tabular}{|c|c|c|}
\hline $\begin{array}{c}3584 \text { faces } \\
8 \text { levels }\end{array}$ & Level 4 & $\begin{array}{c}\text { Original file: } \\
19493 \text { bytes } \\
\text { Compressed } \\
\text { file: } \\
3246 \text { bytes } \\
\\
\text { Ratio: } \\
6.1: 1\end{array}$ \\
\hline $\begin{array}{c}4454 \text { faces } \\
10 \text { levels }\end{array}$ & 306 faces & $\begin{array}{c}\text { Original file : } \\
25894 \text { bytes } \\
\text { Compressed } \\
\text { file: } \\
4062 \text { bytes } \\
\\
\text { Ratio : } \\
6.4: 1\end{array}$ \\
\hline $\begin{array}{l}\text { Original mesh } \\
1916 \text { faces } \\
10 \text { levels }\end{array}$ & 168 faces & $\begin{array}{c}\text { Original file: } \\
9705 \text { bytes } \\
\text { Compressed } \\
\text { file : } \\
1801 \text { bytes } \\
\\
\text { Ratio : } \\
5.4: 1\end{array}$ \\
\hline
\end{tabular}

\section{Conclusion}

We proposed a new scheme allowing to process multiresolution analysis on arbitrary meshes. In sharp contrast with [4] where a resampling of the original mesh is necessary, our scheme processes directly on the original mesh. The proposed method has many potential applications such as mesh compression, progressive transmission and fast rendering of 3D images.

\section{Acknowledgments:}

The authors are grateful to Prof. E. Rittman and R. Robb for providing the $3 \mathrm{D}$ x-ray angiographic data, and $\mathrm{J}$. Lotjonen for building the meshes.

This work is in the scope of the scientific topics of the GdR-PRC ISIS research group of the French National Center for Scientific research.

\section{References}

[1] Paul S. Heckbert and Michael Garland, Survey of polygonal surface simplification algorithms, School of computer science, Carnegie Mellon University, Pittsburgh. URL:http://www.cs.cmu.edu/afs/cs/user/garland/www/multires/

[2] M. Soucy and D. Laurendau, Multiresolution surface modeling based on hierarchical triangulation, in Computer vision and image understanding, volume 63, No 1, January 1996, pages 1-14.

[3] H. Hoppe, Progressive meshes. Computer Graphics (SIGGRAPH '96 Proceedings), pages 99-108.

URL : http://www.research.microsoft.com/ hoppe/

[4] Michael Lounsbery. Multiresolution Analysis for Surfaces of Arbitrary Topological Type. PhD thesis, Dept. of Computer Science and Engineering, U. of Washington, 1994.

URL : ftp://ftp.cs.washington.edu/pub/graphics/LounsPhd.ps.Z

[5] Matthias Eck, Tony DeRose, Tom Duchamp, Hugues Hoppe, Michael Lounsbery, and Werner Stuetzle. Multiresolution Analysis of Arbitrary Meshes. Technical Report \#95-01-02, January 1995.

URL : http://www.cs.washington.edu/research/projects/grail2/ www/pub/abstracts.html

[6] Wim SWELDENS, The Lifting Scheme : A Custom-Design Construction of Biorthogonal Wavelets, Applied and Computational Harmonic Analysis, April 1996, Vol. 3, No. 2, pp.186-200.

[7 ] J. Lotjonen,, P.J. Reissman, I.E. Magnin, J. Nenonen, and T. Katila, A triangulation method of an arbitrary point set for biomagnetic problem, IEEE transactions on magnetics, Vol 34, No 4, July 1998

[8] G. Taubin, J. Rossignac, Geometric compression Through Topological Surgery, IBM Research Report. URL : http://www.research.ibm.com/vrml/binary/ 